Xiao-Hui Wang, Hui-yun Wang, Jia-Zhen Wang, Mei Zhang, Xiao-Qian Chen, Feng-Lan Zhao and Qing-Guo Meng*

\title{
The crystal structure of 3-oxo-urs-12-en-28-oic acid, $\mathrm{C}_{30} \mathrm{H}_{46} \mathrm{O}_{3} \cdot 1 / 6 \mathrm{H}_{2} \mathrm{O}$
}

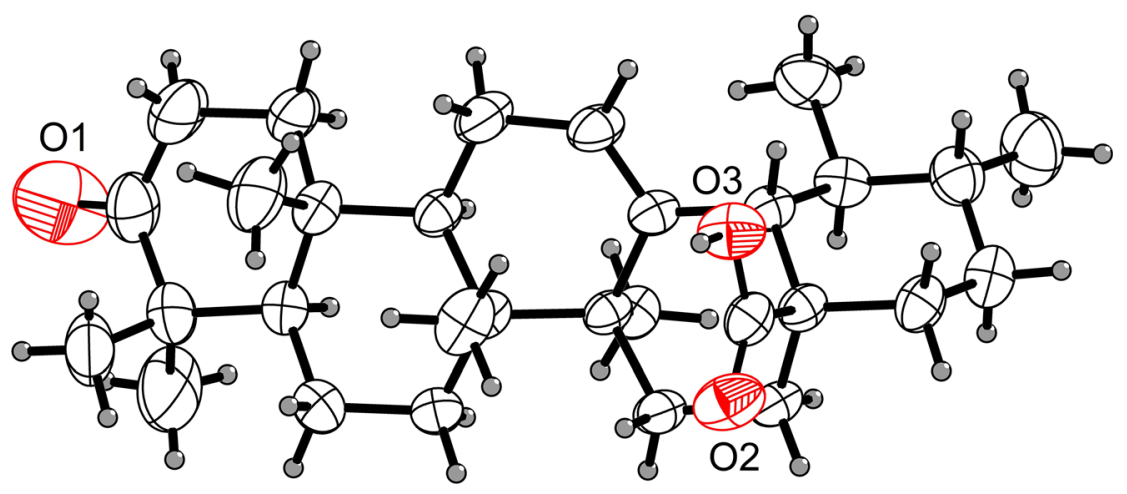

https://doi.org/10.1515/ncrs-2020-0423

Received August 2, 2020; accepted October 16, 2020; published online November 6, 2020

\section{Abstract}

$\mathrm{C}_{30} \mathrm{H}_{46} \mathrm{O}_{3} \cdot 1 / 6 \mathrm{H}_{2} \mathrm{O}$, monoclinic, $\mathrm{C} 2$ (no. 5), $a=$ 29.4307(4) $\AA, b=15.42797(17) \AA, c=18.4667(2) \AA$, $\beta=104.4652(12)^{\circ}, V=8119.11(17) \AA^{3}, Z=12, R_{\mathrm{gt}}(F)=0.0432$, $w R_{\text {ref }}\left(F^{2}\right)=0.1197, T=293(2) \mathrm{K}$.

\section{CCDC no.: 2016692}

One of three independent molecules of the title crystal structure is shown in the Figure. Table 1 contains crystallographic data and Table 2 contains the list of the atoms including atomic coordinates and displacement parameters.

*Corresponding author: Qing-Guo Meng, School of Pharmacy, Collaborative Innovation Center of Advanced Drug Delivery System and Biotech Drugs in Universities of Shandong, Key Laboratory of Molecular Pharmacology and Drug Evaluation (Yantai University), Ministry of Education, Yantai University, Yantai, P. R. China, e-mail: qinggmeng@163.com

Xiao-Hui Wang, Jia-Zhen Wang, Mei Zhang, Xiao-Qian Chen and Feng-Lan Zhao, School of Pharmacy, Collaborative Innovation Center of Advanced Drug Delivery System and Biotech Drugs in Universities of Shandong, Key Laboratory of Molecular Pharmacology and Drug Evaluation (Yantai University), Ministry of Education, Yantai University, Yantai, P. R. China. https://orcid.org/0000-0003-42890163 (X.-H. Wang)

Hui-yun Wang, College of Pharmacy, Jining Medical University, Rizhao, 276826, P. R. China
Table 1: Data collection and handling.

\begin{tabular}{|c|c|}
\hline Crystal: & Colorless block \\
\hline Size: & $0.29 \times 0.24 \times 0.20 \mathrm{~mm}$ \\
\hline Wavelength: & Cu $K \alpha$ radiation (1.54184 ̊̊) \\
\hline$\mu:$ & $0.54 \mathrm{~mm}^{-1}$ \\
\hline Diffractometer, scan mode: & Xcalibur, $\omega$ \\
\hline$\theta_{\max }$, completeness: & $66.8^{\circ}, 99 \%$ \\
\hline$N(h k l)_{\text {measured }}, N(h k l)_{\text {unique }}, R_{\text {int }}:$ & $34,354,12,647,0.029$ \\
\hline Criterion for $I_{\mathrm{obs}}, N\left(h k l_{\mathrm{gt}}\right.$ : & $I_{\mathrm{obs}}>2 \sigma\left(I_{\mathrm{obs}}\right), 11372$ \\
\hline$N(\text { param })_{\text {refined: }}$ & 917 \\
\hline Programs: & $\begin{array}{l}\text { CrysAlis }^{\text {PRO }}[1], \text { Olex2 [2], } \\
\text { SUPERFLIP [3-5], SHELX [6] }\end{array}$ \\
\hline
\end{tabular}

\section{Source of material}

3-Oxo-urs-12-en-28-oic acid was synthesized by oxidation of ursolic acid (UA) with the Sarett reagent. Specifically, the Sarett reagent was added to a solution of UA in dichloromethane (DCM) at room temperature over a period till the brown color persisted. The resulting solution was stirred for further $6 \mathrm{~h}$. The progress of the reaction was monitored by TLC. After completion of the reaction, isopropanol was added and stirred at r.t. for $30 \mathrm{~min}$. The DCM was evaporated in vacuo to yield a black solid, which was purified using silica-gel column chromatography (petroleum ether:ethyl acetate $=20: 1, v / v$ ) to afford a white powder. Suitable crystals were obtained by recrystallization in ethyl acetate solution. 
Table 2: Fractional atomic coordinates and isotropic or equivalent isotropic displacement parameters $\left(\AA^{2}\right)$.

\begin{tabular}{|c|c|c|c|c|c|c|c|c|c|}
\hline \multirow[b]{2}{*}{ Atom } & \multirow[b]{2}{*}{$x$} & \multirow[b]{2}{*}{$y$} & \multirow[b]{2}{*}{$z$} & \multirow[b]{2}{*}{$U_{\text {iso }}{ }^{*} / U_{\text {eq }}$} & \multirow{2}{*}{$\frac{\text { Atom }}{\mathrm{H} 24 \mathrm{G}}$} & $x$ & \multirow{2}{*}{$y$} & \multirow{2}{*}{$\frac{\boldsymbol{z}}{44782}$} & \multirow{2}{*}{$\frac{U_{\text {iso }}{ }^{*} / U_{\text {eq }}}{0.214^{*}}$} \\
\hline & & & & & & 0.671938 & & & \\
\hline 01 & $0.5867(2)$ & $0.0091(5)$ & $0.6690(3)$ & $0.206(3)$ & $\mathrm{H} 24 \mathrm{H}$ & 0.648328 & -0.027414 & 0.585423 & $0.214^{*}$ \\
\hline 02 & $0.81273(9)$ & $0.5601(2)$ & $0.58312(15)$ & $0.0939(8)$ & H24I & 0.689323 & 0.030138 & 0.631619 & $0.214^{\star}$ \\
\hline 03 & $0.82126(8)$ & $0.5673(2)$ & 0.70414 (13) & $0.0832(7)$ & $\mathrm{C} 25$ & 0.72265 (17) & $0.1732(3)$ & $0.7114(3)$ & $0.1086(15)$ \\
\hline H3 & 0.845625 & 0.541511 & 0.704072 & $0.125^{\star}$ & $\mathrm{H} 25 \mathrm{G}$ & 0.737438 & 0.170223 & 0.670655 & $0.163^{*}$ \\
\hline $\mathrm{C} 1$ & $0.64593(16)$ & $0.2046(3)$ & $0.7422(2)$ & $0.0847(10)$ & $\mathrm{H} 25 \mathrm{H}$ & 0.717037 & 0.115634 & 0.726841 & $0.163^{*}$ \\
\hline $\mathrm{H} 1 \mathrm{~A}$ & 0.617945 & 0.240355 & 0.729465 & $0.102^{\star}$ & $\mathrm{H} 25 \mathrm{I}$ & 0.742767 & 0.203515 & 0.752559 & $0.163^{*}$ \\
\hline H1B & 0.663778 & 0.220992 & 0.791858 & $0.102^{*}$ & $\mathrm{C} 26$ & $0.75182(11)$ & $0.3422(3)$ & $0.6267(2)$ & $0.0842(10)$ \\
\hline $\mathrm{C} 2$ & $0.6315(2)$ & $0.1089(3)$ & $0.7428(3)$ & $0.1146(17)$ & $\mathrm{H} 26 \mathrm{G}$ & 0.761864 & 0.362608 & 0.584149 & $0.126^{*}$ \\
\hline $\mathrm{H} 2 \mathrm{~A}$ & 0.658852 & 0.075973 & 0.768576 & $0.137^{\star}$ & $\mathrm{H} 26 \mathrm{H}$ & 0.757866 & 0.281143 & 0.632920 & $0.126^{\star}$ \\
\hline $\mathrm{H} 2 \mathrm{~B}$ & 0.608486 & 0.104143 & 0.772169 & $0.137^{\star}$ & $\mathrm{H} 26 \mathrm{I}$ & 0.768729 & 0.372342 & 0.670717 & $0.126^{*}$ \\
\hline C3 & $0.61238(15)$ & $0.0684(3)$ & $0.6719(3)$ & 0.0934 (11) & $\mathrm{C} 27$ & $0.63612(9)$ & $0.4818(2)$ & $0.59340(17)$ & $0.0617(7)$ \\
\hline $\mathrm{C} 4$ & $0.62633(18)$ & $0.0975(3)$ & $0.6011(2)$ & $0.0941(12)$ & $\mathrm{H} 27 \mathrm{G}$ & 0.624045 & 0.464180 & 0.634833 & $0.093^{*}$ \\
\hline C5 & $0.64576(12)$ & $0.1921(2)$ & $0.60814(18)$ & $0.0678(8)$ & $\mathrm{H} 27 \mathrm{H}$ & 0.619764 & 0.451455 & 0.549165 & $0.093^{*}$ \\
\hline H5 & 0.617630 & 0.228598 & 0.599046 & $0.081^{*}$ & $\mathrm{H} 27 \mathrm{I}$ & 0.631684 & 0.543072 & 0.585686 & $0.093^{*}$ \\
\hline C6 & 0.66939 (15) & $0.2177(2)$ & $0.5464(2)$ & $0.0802(9)$ & $\mathrm{C} 28$ & $0.79677(10)$ & $0.5790(2)$ & $0.63502(17)$ & $0.0612(7)$ \\
\hline H6A & 0.700742 & 0.193304 & 0.557064 & $0.096^{*}$ & C29 & $0.6608(2)$ & $0.8166(4)$ & 0.7045 & $0.1164(16)$ \\
\hline H6B & 0.651618 & 0.194471 & 0.498942 & $0.096^{*}$ & H29G & 0.634197 & 0.806733 & 0.663189 & $0.175^{\star}$ \\
\hline $\mathrm{C} 7$ & $0.67217(12)$ & $0.3153(2)$ & $0.54113(17)$ & $0.0668(7)$ & $\mathrm{H} 29 \mathrm{H}$ & 0.671565 & 0.875160 & 0.702828 & $0.175^{\star}$ \\
\hline H7A & 0.640574 & 0.338578 & 0.526466 & $0.080^{*}$ & H29I & 0.652009 & 0.807346 & 0.750596 & $0.175^{\star}$ \\
\hline H7B & 0.687709 & 0.329975 & 0.502202 & $0.080^{*}$ & C30 & $0.66287(15)$ & $0.6325(3)$ & $0.7575(2)$ & $0.0904(11)$ \\
\hline C8 & $0.69863(9)$ & 0.35 & & & & & & & $0.136^{\star}$ \\
\hline C9 & $0.68082(10)$ & $0.3223(2)$ & $0.68112(15)$ & $0.0555(6)$ & $\mathrm{H} 30 \mathrm{H}$ & 0.637762 & 0.671421 & 0.759515 & $0.136^{*}$ \\
\hline H9 & 0.648855 & 0.344946 & 0.673465 & $0.067^{\star}$ & $\mathrm{H} 30 \mathrm{Ol}$ & 0.686531 & 0.635082 & 03846 & $0.136^{\star}$ \\
\hline C10 & $0.67558(11)$ & $0.2219(2)$ & $0.68597(18)$ & $0.0661(7)$ & 01B & (9) & $0(2)$ & 0.281 & $0.1010(9)$ \\
\hline C11 & $0.70835(14)$ & $0.3634(3)$ & $0.75465(18)$ & $0.0828(11)$ & O2B & $0.54190(7)$ & 0.33317 (19) & $0.45594(10)$ & $0.0734(6)$ \\
\hline $\mathrm{H} 11 \mathrm{E}$ & 0.689882 & 0.358446 & 0.791221 & $0.099^{\star}$ & 03В & $0.46502(7)$ & 0.33191 (19) & $0.40640(11)$ & $0.0744(6)$ \\
\hline $\mathrm{H} 11 \mathrm{~F}$ & 0.737060 & 0.330730 & 0.773263 & 0.099 * & H3B & 0.464788 & 0.331844 & 0717 & $0.112^{\star}$ \\
\hline C12 & $0.72067(12)$ & $0.4562(2)$ & $0.74834(16)$ & $0.0702(8)$ & & $0.62096(10)$ & $0.7771(2)$ & $0.3542(2)$ & $0.0767(10)$ \\
\hline H12 & 0.735000 & 0.484809 & 0.792481 & $0.084^{\star}$ & H1BA & 0.647531 & 0.749705 & 0.388332 & $0.092^{\star}$ \\
\hline C13 & $0.71313(9)$ & $9(2)$ & $0.68574(14)$ & $0.0537(6)$ & H1BB & 2085 & 0.762033 & 3707 & $0.092^{\star}$ \\
\hline C14 & $0.68919(9)$ & $.46032(18)$ & $0.61035(14)$ & $0.0508(6)$ & $\mathrm{C} 2 \mathrm{~B}$ & $0.62565(11)$ & $0.8759(3)$ & 7 (3) & $0.0932(12)$ \\
\hline C15 & $0.70782(11)$ & $0.5008(2)$ & 0.54577 (15) & $0.0614(7)$ & $\mathrm{H} 2 \mathrm{BA}$ & 0.629581 & 0.890400 & 6018 & $0.112^{\star}$ \\
\hline H15E & 0.736816 & & 0.544320 & $0.074^{\star}$ & & & 0.894924 & 259 & $0.112^{\star}$ \\
\hline H15F & 0.685220 & 0.488969 & 0.498742 & $0.074^{\star}$ & C3B & $0.58429(11)$ & & $2(2)$ & $0.0773(9)$ \\
\hline C16 & $0.71689(10)$ & $0.5977(2)$ & $0.55143(15)$ & $0.0601(7)$ & C4B & $0.53508(10)$ & $0.8917(2)$ & $33(2)$ & $0.0680(8)$ \\
\hline H16E & 0.687273 & 0.628033 & 0.544898 & $0.072^{*}$ & $\mathrm{C} 5 \mathrm{~B}$ & $0.53382(8)$ & 0.79018 (17) & $0.31693(16)$ & $0.0508(6)$ \\
\hline $\mathrm{H} 16 \mathrm{~F}$ & 0.730921 & 0.615634 & & $0.072^{\star}$ & $\mathrm{H} 5 \mathrm{~B}$ & 0.535805 & 0.775022 & 0.266279 & $0.061^{\star}$ \\
\hline C17 & $0.74956(9)$ & $0.6230(2)$ & $0.62708(15)$ & $0.0561(6)$ & C6B & $0.48726(9)$ & $0.7517(2)$ & $0.32311(19)$ & $0.0615(7)$ \\
\hline C18 & $0.72583(9)$ & $0.5979(2)$ & $0.68990(15)$ & $0.0549(6)$ & H6BA & 0.461565 & 0.785991 & 0.293759 & $0.074^{\star}$ \\
\hline H18 & 0.749599 & & 0.737117 & $0.066^{*}$ & H6BB & & 0.753141 & 0.374840 & $0.074^{*}$ \\
\hline C19 & $0.68422(11)$ & $0.6589(2)$ & $0.69336(18)$ & $0.0664(7)$ & C7B & $0.48276(8)$ & 0.65834 (19) & $0.29499(17)$ & $0.0556(6)$ \\
\hline H19 & 0.659969 & 0.652305 & 0.646483 & $0.080^{*}$ & H7BA & 0.481680 & 0.658359 & 0.242059 & $0.067^{\star}$ \\
\hline $\mathrm{C} 20$ & 0.70001 (13) & $0.7542(3)$ & $0.6998(2)$ & $0.0784(9)$ & H7BB & 0.453267 & 0.634803 & 0.300602 & $0.067^{\star}$ \\
\hline $\mathrm{H} 2 \mathrm{O}$ & 0.724955 & & & $0.094^{*}$ & $\mathrm{C} 8 \mathrm{~B}$ & $0.52298(8)$ & $0.59835(17)$ & $0.33584(13)$ & $0.0468(5)$ \\
\hline C21 & $0.72040(14)$ & $0.7786(3)$ & $0.6351(2)$ & $0.0824(9)$ & $\mathrm{C} 9 \mathrm{~B}$ & $0.57078(8)$ & $0.64406(18)$ & $0.34279(14)$ & $0.0476(5)$ \\
\hline $\mathrm{H} 21 \mathrm{E}$ & 0.730882 & 0.838343 & 0.640779 & 0.099* & $\mathrm{H} 9 \mathrm{~B}$ & 0.574751 & 0.645445 & 0.291673 & $0.057^{\star}$ \\
\hline $\mathrm{H} 21 \mathrm{~F}$ & & & & & $\mathrm{C} 10 \mathrm{~B}$ & $0.57543(9)$ & 0.74087 (19) & $0.36895(15)$ & $0.0552(6)$ \\
\hline $\mathrm{C} 22$ & $0.76128(12)$ & $0.7208(2)$ & $0.6307(2)$ & $0.0702(8)$ & C11B & $0.61063(10)$ & $0.5858(2)$ & $0.3860(2)$ & $0.0698(8)$ \\
\hline $\mathrm{H} 22 \mathrm{E}$ & 0.787027 & 0.731529 & 0.674103 & $0.084^{\star}$ & $\mathrm{H} 11 \mathrm{C}$ & 0.639428 & 0.602085 & 0.372967 & $0.084^{\star}$ \\
\hline $\mathrm{H} 22 \mathrm{~F}$ & 0.771793 & 0.736495 & 0.586657 & $0.084^{*}$ & H11D & 0.615063 & 0.596225 & 0.439121 & $0.084^{*}$ \\
\hline $\mathrm{C} 23$ & $0.5831(3)$ & $0.0931(4)$ & $0.5358(4)$ & $0.179(4)$ & C12B & $0.60226(9)$ & $0.4917(2)$ & $0.37120(16)$ & $0.0593(7)$ \\
\hline H23G & 0.562858 & & 0.538260 & & $\mathrm{H} 12 \mathrm{~B}$ & 0.626584 & 0.454661 & 0.393829 & $0.071^{\star}$ \\
\hline $\mathrm{H} 23 \mathrm{H}$ & 0.566652 & 0.039829 & 0.538243 & $0.268^{\star}$ & C13B & $0.56415(9)$ & $0.45500(18)$ & $0.32950(13)$ & $0.0489(6)$ \\
\hline $\mathrm{H} 23 \mathrm{I}$ & 0.592488 & 0.095336 & 0.489651 & $0.268^{\star}$ & C14B & $0.52257(8)$ & $0.51176(16)$ & $0.28929(12)$ & $0.0451(5)$ \\
\hline $\mathrm{C} 24$ & $0.6624(3)$ & $0.0291(3)$ & $0.5896(4)$ & $0.142(2)$ & C15B & $0.47503(9)$ & $0.46460(18)$ & $0.28050(15)$ & $0.0537(6)$ \\
\hline
\end{tabular}

Table 2: (continued) 
Table 2: (continued)

\begin{tabular}{|c|c|c|c|c|c|c|c|c|c|}
\hline Atom & $x$ & $y$ & $z$ & $U_{\text {iso }}{ }^{*} / U_{\text {eq }}$ & Atom & $x$ & $y$ & $z$ & $U_{\text {iso }}{ }^{*} / U_{\text {eq }}$ \\
\hline $\mathrm{H} 15 \mathrm{C}$ & 0.464744 & 0.472816 & 0.325997 & $0.064^{\star}$ & $\mathrm{H} 2 \mathrm{CB}$ & 0.677438 & 0.981642 & 0.176271 & $0.104^{*}$ \\
\hline H15D & 0.451987 & 0.491810 & 0.240058 & $0.064^{*}$ & $\mathrm{C} 3 \mathrm{C}$ & $0.6537(2)$ & $1.0003(3)$ & 0.0645 & $0.1052(14)$ \\
\hline C16B & $0.47576(11)$ & $0.3679(2)$ & $0.26465(15)$ & $0.0591(7)$ & $\mathrm{C} 4 \mathrm{C}$ & 0.62014 (19) & $0.9665(3)$ & $-0.0066(2)$ & $0.0942(12)$ \\
\hline H16C & 0.480601 & 0.359238 & 0.215088 & $0.071^{*}$ & $\mathrm{C} 5 \mathrm{C}$ & $0.62088(12)$ & $0.8650(2)$ & $-0.00414(16)$ & $0.0638(7)$ \\
\hline $\mathrm{H} 16 \mathrm{D}$ & 0.445574 & 0.343028 & 0.265228 & $0.071^{*}$ & $\mathrm{H} 5 \mathrm{C}$ & 0.652207 & 0.849611 & -0.009347 & $0.077^{\star}$ \\
\hline C17B & $0.51444(11)$ & 0.32051 (19) & $0.32204(14)$ & $0.0556(6)$ & $\mathrm{C} 6 \mathrm{C}$ & $0.58735(13)$ & $0.8216(2)$ & $-0.07124(17)$ & $0.0741(9)$ \\
\hline C18B & $0.56276(10)$ & $0.35620(18)$ & $0.31921(14)$ & $0.0544(6)$ & $\mathrm{H} 6 \mathrm{CA}$ & 0.555715 & 0.822060 & -0.064434 & 0.089 * \\
\hline $\mathrm{H} 18 \mathrm{~B}$ & 0.585265 & 0.332419 & 0.363030 & $0.065^{\star}$ & $\mathrm{H} 6 \mathrm{CB}$ & 0.587265 & 0.853588 & -0.116509 & $0.089^{\star}$ \\
\hline C19B & 0.57919 (13) & $0.3246(2)$ & $0.24972(17)$ & $0.0692(8)$ & $\mathrm{C} 7 \mathrm{C}$ & $0.60290(12)$ & $0.7286(2)$ & $-0.07864(15)$ & $0.0631(7)$ \\
\hline H19B & 0.558515 & 0.350779 & 0.205165 & $0.083^{*}$ & $\mathrm{H} 7 \mathrm{CA}$ & 0.633553 & 0.729433 & -0.089271 & $0.076^{\star}$ \\
\hline $\mathrm{C} 20 \mathrm{~B}$ & 0.57500 (19) & $0.2251(2)$ & $0.2412(2)$ & $0.0909(13)$ & $\mathrm{H} 7 \mathrm{CB}$ & 0.581147 & 0.701801 & -0.120931 & $0.076^{*}$ \\
\hline $\mathrm{H} 20 \mathrm{~B}$ & 0.596529 & 0.199173 & 0.285020 & $0.109^{\star}$ & $\mathrm{C} 8 \mathrm{C}$ & $0.60562(8)$ & $0.67255(18)$ & $-0.00883(13)$ & $0.0481(5)$ \\
\hline C21B & $0.5265(2)$ & $0.1947(2)$ & $0.2400(2)$ & $0.0948(13)$ & $\mathrm{CgC}$ & $0.63137(8)$ & 0.72287 (18) & 0.06254 (13) & $0.0481(5)$ \\
\hline $\mathrm{H} 21 \mathrm{C}$ & 0.525310 & 0.132054 & 0.235467 & $0.114^{\star}$ & $\mathrm{H} 9 \mathrm{C}$ & 0.664287 & 0.724597 & 0.060335 & $0.058^{\star}$ \\
\hline H21D & 0.504577 & 0.218718 & 0.196451 & $0.114^{\star}$ & $\mathrm{C} 10 \mathrm{C}$ & $0.61752(9)$ & $0.8205(2)$ & $0.06941(15)$ & $0.0554(6)$ \\
\hline $\mathrm{C} 22 \mathrm{~B}$ & $0.51171(16)$ & $0.2212(2)$ & $0.30996(18)$ & $0.0760(9)$ & $\mathrm{C} 11 \mathrm{C}$ & $0.63144(12)$ & $0.6691(2)$ & $0.13221(15)$ & $0.0636(7)$ \\
\hline $\mathrm{H} 22 \mathrm{C}$ & 0.531876 & 0.192839 & 0.353045 & $0.091^{*}$ & $\mathrm{H} 11 \mathrm{~A}$ & 0.656685 & 0.689191 & 0.173228 & $0.076^{*}$ \\
\hline$H 22 D$ & 0.479796 & 0.201921 & 0.305877 & $0.091^{*}$ & $\mathrm{H} 11 \mathrm{~B}$ & 0.602127 & 0.678748 & 0.145998 & $0.076^{*}$ \\
\hline C23B & $0.50085(12)$ & $0.9262(2)$ & $0.2475(2)$ & $0.0823(10)$ & $\mathrm{C} 12 \mathrm{C}$ & $0.63733(9)$ & $0.5737(2)$ & $0.12191(14)$ & $0.0548(6)$ \\
\hline$H 23 D$ & 0.506217 & 0.896874 & 0.204512 & $0.123^{\star}$ & $\mathrm{H} 12 \mathrm{C}$ & 0.640257 & 0.538955 & 0.163990 & $0.066^{*}$ \\
\hline $\mathrm{H} 23 \mathrm{E}$ & 0.505710 & 0.987295 & 0.243032 & $0.123^{*}$ & $\mathrm{C} 13 \mathrm{C}$ & $0.63878(8)$ & $0.53371(18)$ & $0.05877(13)$ & $0.0466(5)$ \\
\hline $\mathrm{H} 23 \mathrm{~F}$ & 0.469205 & 0.915993 & 0.250516 & $0.123^{*}$ & C14C & $0.63410(8)$ & $0.58604(18)$ & $-0.01343(13)$ & $0.0466(5)$ \\
\hline C24B & $0.52183(15)$ & $0.9326(3)$ & $0.3865(3)$ & $0.0970(13)$ & $\mathrm{C} 15 \mathrm{C}$ & $0.60845(10)$ & 0.53319 (19) & $-0.08299(14)$ & $0.0528(6)$ \\
\hline$H 24 D$ & 0.491303 & 0.912688 & 0.388685 & $0.145^{\star}$ & $\mathrm{H} 15 \mathrm{~A}$ & 0.574908 & 0.538001 & -0.088342 & $0.063^{*}$ \\
\hline $\mathrm{H} 24 \mathrm{E}$ & 0.521457 & 0.994612 & 0.381799 & $0.145^{\star}$ & $\mathrm{H} 15 \mathrm{~B}$ & 0.615247 & 0.559169 & -0.126956 & $0.063^{*}$ \\
\hline $\mathrm{H} 24 \mathrm{~F}$ & 0.544481 & 0.916040 & 0.431404 & $0.145^{\star}$ & $\mathrm{C} 16 \mathrm{C}$ & $0.62120(10)$ & 0.43798 (19) & $-0.08125(15)$ & $0.0547(6)$ \\
\hline$C 25 B$ & $0.57787(15)$ & $0.7520(3)$ & 0.45288 (19) & $0.0862(10)$ & $\mathrm{H} 16 \mathrm{~A}$ & 0.653508 & 0.432250 & -0.084184 & $0.066^{\star}$ \\
\hline$H 25 D$ & 0.595310 & 0.803457 & 0.471237 & $0.129^{\star}$ & H16B & 0.601417 & 0.409439 & -0.124604 & $0.066^{*}$ \\
\hline $\mathrm{H} 25 \mathrm{E}$ & 0.593148 & 0.702575 & 0.479977 & $0.129^{*}$ & $\mathrm{C} 17 \mathrm{C}$ & 0.61534 (9) & $0.39313(18)$ & $-0.01042(14)$ & $0.0528(6)$ \\
\hline $\mathrm{H} 25 \mathrm{~F}$ & 0.546674 & 0.756898 & 0.459642 & $0.129^{*}$ & $\mathrm{C} 18 \mathrm{C}$ & $0.64763(9)$ & $0.43686(19)$ & 0.05929 (15) & $0.0523(6)$ \\
\hline C26B & $0.51576(12)$ & $0.5764(2)$ & $0.41406(16)$ & $0.0665(8)$ & $\mathrm{H} 18 \mathrm{C}$ & 0.638108 & 0.413759 & 0.102702 & $0.063^{\star}$ \\
\hline$H 26 D$ & 0.488777 & 0.539683 & 0.408583 & $0.100^{*}$ & $\mathrm{C} 19 \mathrm{C}$ & $0.70031(10)$ & $0.4120(2)$ & $0.07052(18)$ & $0.0688(8)$ \\
\hline $\mathrm{H} 26 \mathrm{E}$ & 0.511025 & 0.628951 & 0.439025 & $0.100^{\star}$ & $\mathrm{H} 19 \mathrm{C}$ & 0.711065 & 0.436300 & 0.028665 & $0.083^{*}$ \\
\hline $\mathrm{H} 26 \mathrm{~F}$ & 0.543010 & 0.546940 & 0.443041 & $0.100^{*}$ & C20C & 0.70609 (13) & $0.3134(3)$ & $0.0691(2)$ & 0.0835 (11) \\
\hline C27B & $0.52876(10)$ & $0.52852(19)$ & 0.20915 (13) & $0.0548(6)$ & $\mathrm{H} 20 \mathrm{C}$ & 0.695616 & 0.289301 & 0.111276 & $0.100^{*}$ \\
\hline $\mathrm{H} 27 \mathrm{D}$ & 0.560363 & 0.546872 & 0.212276 & $0.082^{\star}$ & $\mathrm{C} 21 \mathrm{C}$ & $0.67651(14)$ & $0.2738(3)$ & $-0.0016(2)$ & 0.0857 (11) \\
\hline H27E & 0.507323 & 0.572923 & 0.185388 & $0.082^{\star}$ & $\mathrm{H} 21 \mathrm{~A}$ & 0.680729 & 0.211367 & 0.000406 & $0.103^{*}$ \\
\hline $\mathrm{H} 27 \mathrm{~F}$ & 0.522416 & 0.476104 & 0.180376 & $0.082^{\star}$ & $\mathrm{H} 21 \mathrm{~B}$ & 0.686904 & 0.295380 & -0.044055 & $0.103^{*}$ \\
\hline C28B & $0.50774(10)$ & 0.33101 (19) & $0.40069(14)$ & $0.0549(6)$ & $\mathrm{C} 22 \mathrm{C}$ & $0.62449(13)$ & $0.2948(2)$ & $-0.0123(2)$ & 0.0704 (8) \\
\hline C29B & 0.5894 & $0.1922(3)$ & $0.1712(3)$ & $0.131(2)$ & $\mathrm{H} 22 \mathrm{~A}$ & 0.613190 & 0.266771 & 0.026899 & $0.084^{\star}$ \\
\hline$H 29 D$ & 0.569430 & 0.218049 & 0.127367 & $0.197^{\star}$ & $\mathrm{H} 22 \mathrm{~B}$ & 0.606956 & 0.271531 & -0.059855 & $0.084^{\star}$ \\
\hline $\mathrm{H} 29 \mathrm{E}$ & 0.586204 & 0.130332 & 0.168194 & $0.197^{\star}$ & C23C & $0.6372(3)$ & $0.9963(4)$ & $-0.0741(3)$ & $0.146(3)$ \\
\hline $\mathrm{H} 29 \mathrm{~F}$ & 0.621390 & 0.207911 & 0.174615 & $0.197^{\star}$ & $\mathrm{H} 23 \mathrm{~A}$ & 0.664807 & 0.964324 & -0.076263 & $0.220^{*}$ \\
\hline C30B & 0.62901 (15) & $0.3562(3)$ & $0.2549(2)$ & $0.0923(12)$ & $\mathrm{H} 23 \mathrm{~B}$ & 0.644382 & 1.057004 & -0.069634 & $0.220^{*}$ \\
\hline H30D & 0.629253 & 0.418355 & 0.252853 & $0.139^{\star}$ & $\mathrm{H} 23 \mathrm{C}$ & 0.613032 & 0.986147 & -0.119016 & 0.220 * \\
\hline $\mathrm{H} 30 \mathrm{E}$ & 0.639808 & 0.333010 & 0.213956 & $0.139^{\star}$ & C24C & $0.5720(2)$ & $1.0073(4)$ & $-0.0115(4)$ & $0.138(2)$ \\
\hline $\mathrm{H} 30 \mathrm{~F}$ & 0.649341 & 0.337082 & 0.301290 & $0.139^{\star}$ & $\mathrm{H} 24 \mathrm{~A}$ & 0.549252 & 0.982564 & -0.052887 & $0.207^{*}$ \\
\hline $01 \mathrm{C}$ & $0.6814(2)$ & $1.0565(4)$ & $0.0633(3)$ & $0.190(3)$ & H24B & 0.573691 & 1.068719 & -0.018592 & $0.207^{\star}$ \\
\hline $\mathrm{O} 2 \mathrm{C}$ & $0.53260(7)$ & $0.39302(18)$ & $-0.06581(10)$ & $0.0709(6)$ & $\mathrm{H} 24 \mathrm{C}$ & 0.562760 & 0.996114 & 0.034001 & $0.207^{\star}$ \\
\hline $\mathrm{H} 2 \mathrm{C}$ & 0.507221 & 0.385396 & -0.055970 & $0.106^{*}$ & $\mathrm{C} 25 \mathrm{C}$ & $0.56941(11)$ & $0.8324(3)$ & $0.0877(2)$ & $0.0766(9)$ \\
\hline $\mathrm{O3C}$ & $0.55522(7)$ & 0.40157 (18) & $0.05711(11)$ & $0.0691(6)$ & $\mathrm{H} 25 \mathrm{~A}$ & 0.544909 & 0.829813 & 0.042354 & $0.115^{\star}$ \\
\hline $\mathrm{C} 1 \mathrm{C}$ & $0.65497(12)$ & $0.8612(2)$ & $0.13312(17)$ & $0.0681(8)$ & $\mathrm{H} 25 \mathrm{~B}$ & 0.568552 & 0.887660 & 0.111262 & $0.115^{\star}$ \\
\hline $\mathrm{H} 1 \mathrm{CA}$ & 0.651996 & 0.837028 & 0.180221 & $0.082^{\star}$ & $\mathrm{H} 25 \mathrm{C}$ & 0.564902 & 0.787146 & 0.120911 & $0.115^{*}$ \\
\hline $\mathrm{H} 1 \mathrm{CB}$ & 0.685728 & 0.845543 & 0.127055 & $0.082^{\star}$ & $\mathrm{C} 26 \mathrm{C}$ & $0.55512(9)$ & $0.6495(2)$ & -0.00511 (19) & $0.0658(8)$ \\
\hline $\mathrm{C} 2 \mathrm{C}$ & $0.65174(16)$ & $0.9599(3)$ & $0.1368(2)$ & $0.0863(10)$ & $\mathrm{H} 26 \mathrm{~A}$ & 0.556273 & 0.612664 & 0.037199 & $0.099 *$ \\
\hline $\mathrm{H} 2 \mathrm{CA}$ & 0.622572 & 0.975939 & 0.148669 & $0.104^{\star}$ & H26B & 0.539353 & 0.619882 & -0.050088 & $0.099^{\star}$ \\
\hline
\end{tabular}

Table 2: (continued) 
Table 2: (continued)

\begin{tabular}{lrrrr}
\hline Atom & $\boldsymbol{x}$ & $\boldsymbol{y}$ & $\boldsymbol{z}$ & $\boldsymbol{U}_{\text {iso }} \boldsymbol{U}_{\text {eq }}$ \\
\hline H26C & 0.538385 & 0.701687 & -0.000139 & $0.099^{\star}$ \\
C27C & $0.68417(9)$ & $0.6046(2)$ & $-0.02168(16)$ & $0.0578(6)$ \\
H27A & 0.702940 & 0.627728 & 0.024378 & $0.087^{*}$ \\
H27B & 0.682677 & 0.645765 & -0.061160 & $0.087^{*}$ \\
H27C & 0.697998 & 0.551687 & -0.033379 & $0.087^{*}$ \\
C28C & $0.56441(9)$ & $0.39821(18)$ & $-0.00600(14)$ & $0.0493(5)$ \\
C29C & $0.75723(18)$ & $0.2862(5)$ & $0.0781(4)$ & $0.130(2)$ \\
H29A & 0.768697 & 0.310667 & 0.038248 & $0.195^{*}$ \\
H29B & 0.759133 & 0.224177 & 0.076484 & $0.195^{*}$ \\
H29C & 0.775937 & 0.306812 & 0.125222 & $0.195^{*}$ \\
C30C & $0.72983(12)$ & $0.4517(3)$ & $0.1424(2)$ & $0.0923(12)$ \\
H30A & 0.727594 & 0.513758 & 0.139260 & $0.138^{*}$ \\
H30B & 0.761986 & 0.434576 & 0.149252 & $0.138^{*}$ \\
H30C & 0.718545 & 0.431914 & 0.183993 & $0.138^{*}$ \\
\hline
\end{tabular}

\section{Experimental details}

All hydrogen atoms were placed in idealized positions with the carrier atom $-\mathrm{H}$ distances $=0.93 \AA$ for alkene, $0.96 \AA$ for methyl, $0.97 \AA$ for methylene and $0.98 \AA$ for methine. The $U_{\text {iso }}$ values of the hydrogen atoms of methyl groups were set to $1.5 U_{\text {eq }}$ and the $U_{\text {iso }}$ values of all other hydrogen atoms were set to $1.2 U_{\text {eq }}(\mathrm{C})$. The absolute configuration was derived from the synthesis. The solvent molecule was presumed to be two water molecule on the basis of the $43 \AA^{3}$ voids.

\section{Comment}

Ursolic acid (UA), a pentacyclic triterpenoid compound, is widely distributed in food products such as apples, basil, as well as in more than 120 plant species, many of which are applied as medicinal plants in traditional formulations [7]. UA has been reported to have multiple pharmacological activities like anti-inflammatory [8], antitumor [9], antioxidative [10], antiviral [11]. The pentacyclic triterpene system of title compound molecule consists of condensed six-membered rings with a ketone functional group at C-3 and a carboxyl moiety at $\mathrm{C}-28$. It has been reported that 3-oxo-ursolic acid derivatives exhibit significant activity in inhibiting the production of nitric oxide induced by interferon-y in mouse macrophages [12, 13]. And other study also showed that its derivatives more potently inhibit the growth of MCF-7 and THP-1 cancer cell lines [14]. Accordingly, the title compound was synthesized according to reported procedures [15]. In the molecule of the title compound, bond lengths and angles within the sixmembered rings are similar to those given in the literature for tetracyclic triterpeneoids [16-18].

Single-crystal structure analysis indicated that there are three molecules in the asymmetric unit of the title crystal structure. All cyclohexane rings are in the usually observed chair conformation, cyclohexene is in a conformation intermediate between sofa and half-chair: the C8 and $\mathrm{C} 9$ atoms deviate in different directions with respect to the planar $\mathrm{C} 11-\mathrm{C} 12=\mathrm{C} 13-\mathrm{C} 14$ fragment by 0.66 and $0.12 \AA$, respectively, in one molecule, by 0.70 and $0.84 \AA$ in the second molecule and by 0.66 and $0.13 \AA$ in the third molecule.

Acknowledgments: X-ray data were collected at Institute of Medical Biotechnology, Chinese Academy of Medical Sciences \& Peking Union Medical College.

Author contribution: All the authors have accepted responsibility for the entire content of this submitted manuscript and approved submission.

Research funding: This work was supported by Science and Technology Innovation Development Plan of Yantai (No. 2020XDRH105) and the National Natural Science Foundation of China (No. 81473104 and 81773563).

Conflict of interest statement: The authors declare no conflicts of interest regarding this article.

\section{References}

1. Rigaku Oxford Diffraction: CrysAlis ${ }^{P R O}$; Rigaku Corporation: Oxford, UK, 2018.

2. Dolomanov O. V., Bourhis L. J., Gildea R. J., Howard J. A. K., Puschmann H. Olex2: a complete structure solution, refinement and analysis program. J. Appl. Cryst. 2009, 42, 339-341.

3. Palatinus L., Chapuis G. SUPERFLIP - a computer program for the solution of crystal structures by charge flipping in arbitrary dimensions. J. Appl. Cryst. 2007, 40, 786-790.

4. Palatinus L., van der Lee A. Symmetry determination following structure solution in P1. J. Appl. Cryst. 2008, 41, 975-984.

5. Palatinus L., Prathapa S. J., van Smaalen S. EDMA: a computer program for topological analysis of discrete electron densities. J. Appl. Cryst. 2012, 45, 575-580.

6. Sheldrick G. M. Crystal structure refinement with SHELXL. Acta Crystallogr. 2015, C71, 3-8.

7. Wang X. T., Gong Y., Zhou B., Yang J. J., Cheng Y., Zhao J. G., Qi M. Y. Ursolic acid ameliorates oxidative stress, inflammation and fibrosis in diabetic cardiomyopathy rats. Biomed. Pharmacother. 2018, 97, 1461-1467.

8. Wei Z. Y., Chi K. Q., Wang K. S., Wu J., Liu L. P., Piao H. R. Design, synthesis, evaluation, and molecular docking of ursolic acid derivatives containing a nitrogen heterocycle as antiinflammatory agents. Bioorg. Med. Chem. Lett. 2018, 28, 17971803. 
9. Jiang W., Huang R. Z., Zhang J., Guo T., Zhang M. T., Huang X. C., Zhang B., Liao Z. X., Sun J., Wang H. S. Discovery of antitumor ursolic acid long-chain diamine derivatives as potent inhibitors of NF-IkB. Bioorg. Chem. 2018, 79, 265-276.

10. Wang X. T., Gong Y., Zhou B., Yang J. J., Cheng Y., Zhao J. G., Qi M. Y. Ursolic acid ameliorates oxidative stress, inflammation and fibrosis in diabetic cardiomyopathy rats. Biomed. Pharmacother. 2018, 97, 1461-1467.

11. Tohmé M. J., Giménez M. C., Peralta A., Colombo M. I., Delgui L. R. Ursolic acid: a novel antiviral compound inhibiting rotavirus infection in vitro. Int. J. Antimicrob. Agents 2019, 54, 601-609.

12. Honda T., Gribble G. W., Suh N., Finlay H. J., Rounds B. V., Bore L., Favaloro F. G., Wang Y. P., Sporn M. B. Novel synthetic oleanane and ursane triterpenoids with various enone functionalities in ring a as inhibitors of nitric oxide production in mouse macrophages. J. Med. Chem. 2000, 41, 1866-1877.

13. Honda T., Rounds B. V., Bore L., Finlay H. J., Favaloro F. G. Jr., Suh N., Wang Y. P., Sporn M. B., Gribble G. W. Synthetic oleanane and ursane triterpenoids with modified rings $A$ and $C$ : a series of highly active inhibitors of nitric oxide production in mouse macrophages. J. Med. Chem. 2000, 41, 4233-4246.
14. Wang W. Z., Lei L., Liu Z., Wang H. B., Meng Q. G. Design, synthesis, and biological evaluation of novel nitrogen heterocycle-containing ursolic acid analogs as antitumor agents. Molecules 2019, 41, 877.

15. Zhao R. L., Wang H. Y., Luan M. Z., Zheng X., Zhao F. L., Meng Q. G. Crystal structure of $(3 R, 5 R, 8 R, 9 R, 10 R, 12 R, 13 R, 14 R)$ 4,4,8,10,14-pentamethyl-17-((R)-2,6,6-trimethyltetrahydro- $2 \mathrm{H}$ pyran-2-yl) hexadecahydro-1H-cyclopenta[a]phenanthrene3,12-diol, $\mathrm{C}_{30} \mathrm{H}_{52} \mathrm{O}_{3}$. Z. Kristallogr. NCS 2020, 235, 129-131.

16. Liu J., Xu Y. R., Yang J. J., Wang W. Z., Zhang J. Q., Zhang R. M., Meng Q. G. Discovery, semisynthesis, biological activities, and metabolism of ocotillol-type saponins. J. Ginseng Res. 2017, 41, 373-378.

17. Wang C. M., Liu J., Deng J. Q., Wang J. Z., Weng W. Z., Chu H. X., Meng Q. G. Advances in the chemistry, pharmacological diversity, and metabolism of 20(R)-ginseng saponins. J. Ginseng Res. 2020, 44, 14-23.

18. Zhang J. Q., Xu Y. R., Li H. X., Zhao F. L., Wang C. M., Liu Z., Liu P., Liu Y. N., Meng Q. G., Zhao F. Synthesis and in vitro antiinflammatory activity of C20 epimeric ocotillol-type triterpenes and protopanaxadiol. Planta Med. 2019, 85, 292-301. 